\title{
Investigação do efeito do tempo de exposição à temperatura ambiente e ao tempo de estocagem de um filme adesivo estrutural de resina epoxídica
}

\section{Investigation of the effect of exposure time at room temperature in a structural epoxy resin adhesive film}

\author{
Ana Carolina Teixeira Neves da Silva ${ }^{1}$, Fernanda Guilherme ${ }^{1}$, \\ Vanesa Mitchell Ferrari ${ }^{1 *}$ e Paulo Eduardo Ferrari² \\ ${ }^{1}$ Faculdade de Engenharias, Arquitetura e Urbanismo - FEAU, Universidade do Vale do Paraíba - UNIVAP, \\ São José dos Campos, SP, Brasil \\ ${ }^{2}$ Embraer S.A., São José dos Campos, SP, Brasil \\ *vanesa_mitchell@yahoo.com.br
}

\begin{abstract}
Resumo
A exposição de filmes adesivos de resina epoxídica à temperatura ambiente propicia condições que afetam diretamente suas propriedades físicas. Para o adesivo estrutural AF191, de aplicação no setor aeronáutico, isto pode ser confirmado pela súbita perda de pegajosidade ("tack"). Sendo assim, o comportamento do sistema de resina escolhido foi estudado experimentalmente, expondo-se este ao ambiente de laboratório a intervalos pré-determinados de tempo e a diferentes períodos de armazenamento $\left(-18^{\circ} \mathrm{C}\right)$. O efeito da exposição foi analisado por meio de ensaios fisico-químicos baseados em normas internacionais complementados por testes empíricos não normatizados que visaram observar alguns comportamentos especificos da resina, como capacidade de fluxo e solubilidade. A partir dos resultados, foi possível observar alterações no comportamento fisico-químico, que afetam as condições de processabilidade do adesivo, como a capacidade de fluxo do adesivo quando aquecido, redução da aderência e da solubilidade em solvente orgânico. Estas alterações sugerem que houve um possível aumento no número de reticulações da matriz polimérica à temperatura ambiente.Porém este fato não foi comprovadopelo método de análise térmica utilizado (calorimetria exploratória diferencial - DSC), onde não foi observado alteração significativa da entalpia de cura ao longo do período de exposição. Isso pode ser atribuído a uma taxa de cura muito baixa da matriz ou apenas pela falta sensibilidade do método. A mudança de comportamento pode estar associada ao processo de plastificação da matriz polímérica causado pela absorção de umidade devido ao caráter higroscópico da matriz epoxídica, que pode ser verificado no aumento progressivo do ensaio de teor de voláteis. O período de estocagem mostrou que não houve alteração significativa das características avaliadas do adesivo.
\end{abstract}

Palavras-chaves: adesivo, resina epoxídica, cura, fluidez, solubilidade.

\begin{abstract}
The exposure of epoxy based adhesive films to room temperature provides conditions that directly affects their physical properties. For structural adhesive AF191, application in the aerospace industry, this can be confirmed by the sudden loss of tack. Therefore, the behavior of the chosen resin system was experimentally studied, by means of the exposure to laboratory environment at pre determinated time interval and different storage periods $\left(-18^{\circ} \mathrm{C}\right)$. The effect of the exposure was evaluated by means of physicochemical tests based on international standards, being complimented by not normalized empirical tests that aimed to observe some specific behavior of the resin, like flow and solubility. From the results, it was possible to observe changes in the physicochemical behavior that affects the adhesive processability conditions such as adhesive flow capacity, self adhesiveness reduction and solubility to organic solvent. These changes suggest that there was a possible increase in the number of crosslinking of the polymer matrix at room temperature. This fact however was not proven by the used thermal analysis method (differential scanning calorimetry - DSC), where since there was no significant change in cure enthalpy was observed during the exposure period. This can be attributed to a very low curing rate of the matrix or just low sensitivity of the method. The change in behavior can be related to the lamination process the polymer matrix caused by moisture absorption due to the hygroscopic nature of the epoxy matrix, which can be found in the progressive increase in the volatile content test. The storage period was efficient indicating no significant change in the characteristics evaluated the adhesive.
\end{abstract}

Keywords: adhesive, epoxy resin, cure, flow, solubility. 


\section{Introdução}

O termo adesivo é utilizado para descrever a substância capaz de manter materiais unidos pelo contato entre superfícies. $\mathrm{O}$ adesivo estrutural é definido como agente de ligação requerido para transferir cargas necessárias entre superfícies (aderentes) expostas ao ambiente de trabalho típico para a estrutura envolvida ${ }^{[1]}$.

$\mathrm{O}$ termo adesivo estrutural costuma ser utilizado para definir um adesivo de resistência elevada de forma a se atingir o sucesso da montagem em seu ambiente operacional com alto grau de confiabilidade, como os filmes epoxídicos e acrílicos. Já os adesivos não estruturais, como a cola de madeira e selantes, possuem menor resistência, e/ou geralmente são usados para fixação temporária ${ }^{[2]}$.

Atualmente, existem diversas técnicas utilizadas para unir duas superfícies como, por exemplo, a soldagem, prendedores mecânicos (rebites, parafusos, etc) e colagem.

A utilização dos adesivos minimiza os efeitos de concentração de tensão, aumenta a flexibilidade da peça e age como vedação a intempéries. Na área de manufatura, a utilização dos adesivos estruturais para colagem elimina a necessidade de furos, reduz o peso e o custo total do processo (Figura 1). Normalmente, a escolha pelo processo e o uso de adesivos pode envolver os estudos preliminares de desempenho, da capacidade de produção, de custo e confiabilidade $^{[4]}$. Os filmes adesivos são, na atualidade, os materiais que despertam maior interesse nos estudos de propriedades e na evolução da técnica denominada colagem ${ }^{[3]}$.

Os filmes adesivos estruturais de resina epoxídica, largamente utilizados para aplicações na indústria aeronáutica, são geralmente comercializados como filmes finos e contínuos, que devem ser armazenados sob refrigeração (geralmente $-18^{\circ} \mathrm{C}$ ). Estes são preferidos aos adesivos líquidos e pastas devido à sua uniformidade e reduzido conteúdo de vazios. Os filmes adesivos possuem agentes que requerem alta temperatura de cura e os mantêm relativamente estáveis à temperatura ambiente por períodos curtos de tempo ${ }^{[5]}$.

As resinas epoxídicas são preferidas porque propiciam a produção de filmes adesivos que, depois de curados, apresentam ótima resistência a solventes, combustíveis e lubrificantes. São amplamente utilizados na indústria aeronáutica, tanto na colagem de materiais metálicos quanto compósitos, devido à diversidade de suas propriedades, garantindo elevada relação entre custo, peso e resistência química a diversos ambientes operacionais, além de excelentes propriedades térmicas, físicas e mecânicas ${ }^{[1,5]}$.

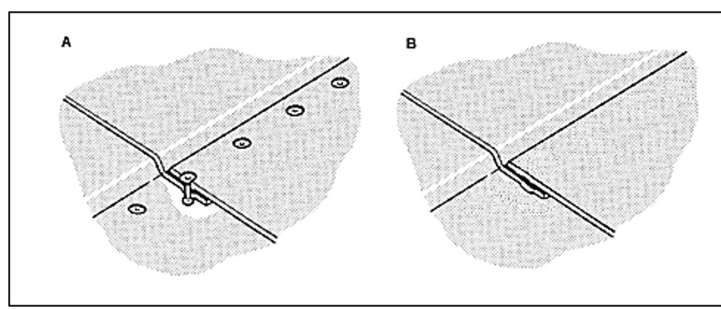

Figura 1. Esquematização de uma junção de peças através de (A) rebites, (B) adesivos estruturais ${ }^{[3]}$.
Os filmes adesivos epoxídicos apresentam aderência à maioria dos materiais sólidos, independentemente da forma, espessura ou de suas propriedades físicas. Apesar de apresentarem custo elevado em nível de matériaprima, proporcionam grande redução de peso e eliminam operações posteriores de acabamento, além da facilidade na sua montagem.

Os adesivos podem ser produzidos em várias formas: soluções à base de solventes, emulsão aquosa, filme suportado ou filme não suportado ${ }^{[4]}$. No processo de fabricação do filme adesivo em estudo, a resina em sua fase líquida impregna totalmente fibras de poliésteres, poliamidas (nylon) ou fibras de vidro. Adesivos com este tipo de construção facilitam o manuseio antes da cura e promovem melhor controle de fluxo de adesivo durante esta, auxiliando o controle de espessura da área de colagem ${ }^{[5]}$.

O processo de fabricação dos filmes adesivos é feito de forma contínua e altamente automatizada, onde as fibras de suporte, em forma de uma manta fina, são depositadas sobre um filme de resina (matriz) e passada entre mandris que regulam a distribuição e homogeneidade da resina enquanto fluída. Esse sistema é então passado em regiões de aquecimento para que a resina atinja um estagio de polimerização intermediária, onde esta não escorre mais, porém, não está completamente curada, denominado estágio B. Após essa etapa, o material é coberto por filme plástico protetivo de polietileno e silicone que servem de separador e como proteção contra contaminação e umidade. Finalmente, os filmes são enrolados em um suporte e armazenados em forma de bobinas ${ }^{[6,7]}$.

No momento da utilização, o filme adesivo é retirado do armazenamento, descongelado até a temperatura ambiente ainda na embalagem, desembalado e cortado no formato desejado, sendo o plástico de proteção retirado no momento da aplicação ${ }^{[3]}$. A forma de filme garante um peso ideal e proporções controladas e exatas de resina e catalisador no adesivo.

Durante o ciclo de aquecimento, o filme se liquefaz e flui o suficiente para molhar as superfícies a serem coladas, permitindo a remoção do ar retido e passando pelo processo de cura, quando se transformam em um sólido infusível ${ }^{[8]}$.

No processo de cura ocorre a formação de ligações cruzadas covalentes entre as cadeias moleculares adjacentes. Estas ligações ancoram as cadeias entre si para resistir aos movimentos vibracionais e rotacionais a altas temperaturas. Apenas o aquecimento até temperaturas excessivas causará a quebra destas ligações onde ocorrerá a degradação do polímero $^{[8]}$.

O tempo de armazenamento ("shelflife") para este tipo de material, que é considerado perecível, é limitado pelo prazo de validade do material em condições de estocagem recomendadas (geralmente $-18^{\circ} \mathrm{C}$ ), com o intuito de preservar ao máximo as propriedades químicas e físicas adequadas ao seu uso, contabilizado pelo período de tempo a partir da sua produção. Este tempo pode variar de poucos dias a mais de um ano, dependendo da natureza do material ou do seu manuseio ${ }^{[2]}$. Outro controle utilizado para este tipo de material é o tempo acumulado do material quando retirado do ambiente de armazenamento e exposto ao ambiente de produção, ("out time"). 
Nos últimos anos, vários artigos relataram o envelhecimento físico em resinas epoxídicas ${ }^{[9]}$. A influência do envelhecimento físico nas propriedades viscoelásticas das resinas baseadas no DGEBA foi bastante estudada por McKenna e colaboradores, pois esta resina serve como modelo no estudo das estruturas reticuladas ${ }^{[10]}$. As alterações estruturais produzidas devido ao envelhecimento físico dependem do tempo e da temperatura e evidenciam o comportamento dos materiais termofixos a temperaturas abaixo da transição vítrea sendo, normalmente, o resultado do relaxamento lento do material vítreo a partir do seu estado inicial de não equilíbrio termodinâmico para o seu estado final de equilíbrio ${ }^{[9]}$. Todas as propriedades que dependem do volume específico (ou do volume livre) se alteram durante este processo e, portanto, o envelhecimento físico possui importância prática na determinação das propriedades de engenharia destes materiais ${ }^{[10]}$.

No trabalho de Kim e co-autoresfoi investigada a influência das condições ambientais às quais o prepreg de resina epoxídica é submetido antes do processo de cura para materiais curados fora da autoclave. Verificou-se por meio de análise MDSC e reometria que a exposição do material à temperaturaambiente alterou o grau inicial de cura da resina, bem como a evolução da taxa de cura e a viscosidade a temperaturas elevadas. Por meio do acompanhamento da evolução das propriedades dielétricas da resina por análise DEA, em condições ambientee a temperatura constante, foram desenvolvidas correlações eficazes para se obter o grau de cura e viscosidade. Comprova-se a eficácia do método de acompanhamento das propriedades dielétricas para o estudo do processo de cura, identificando propriedades e transições da resina e, além disso, prevendo a influência das condições ambientais no processo de polimerização ${ }^{[11]}$.

$\mathrm{O}$ envelhecimento dos adesivos pode ser relacionado com a temperatura e umidade, e mesmo à exposição à luz. Estes efeitos são sempre prejudiciais e a taxa de deterioração depende do tipo e forma da resina ${ }^{[2]}$. A umidade adquirida pela resina epoxídica pode se apresentar de diferentes formas, parte das moléculas de água absorvidas podem se difundir entre as cadeias do polímero, atuar como plastificante aumentando o volume livre e consequentemente, reduzir a temperatura de transição vítrea, outra parte das moléculas de água podem se alojar no interior de microcavidades da matriz polimérica ou se difundir na região da interfase fibra-matriz ${ }^{[12-14]}$, e ainda existe a possibilidade de participar de reações de hidrólise ${ }^{[13]}$. Assim, dependendo da forma que estas moléculas de água se apresentam é possível que causem diferentes efeitos sobre o material ${ }^{[13]}$.

Sales, por meio das técnicas de luminescência e TG, mostrou que poderia ter ocorrido uma plastificação da matriz polimérica devido à presença de água. Embora tenha apresentado bons resultados, a técnica de luminescência é disponível somente em poucos laboratórios especializados, sendo assim, nesse atual trabalho foram usadas técnicas mais convencionais ${ }^{[15]}$.
A exposição de adesivos ao meio ambiente ao longo do tempo provoca alterações de características como aumento da viscosidade e a redução de pegajosidade ("tack") que durante o processo de laminação compromete a capacidade do adesivo de se conformar a raios e contornos ("drape") durante o processo de moldagem. O tempo de gelificação ("gel time") diminui e ocorre perda da capacidade de fluxo durante a cura, prejudicando potencialmente o tempo para a remoção de voláteis durante o processo de cura ${ }^{[16]}$.

Assim, o presente estudo, faz uso de metodologias para investigar o comportamento da resina tanto quando exposta à temperatura ambiente quanto armazenada em freezer por longos períodos, assim como a combinação dessas condições.

\section{Experimental}

O material em estudo é um filme adesivo estrutural de uso aeronáutico, tendo como componente predominante na sua formulação uma matriz de resina epoxídica sustentada por uma trama de fibra de poliamida, denominado comercialmente como AF191, produzido pela empresa 3M.

Para as análises, foram utilizados os seguintes equipamentos:

- Calorímetro Exploratório Diferencial, DSC Perkin Elmer - Modelo Pyris1;

- Equipamento de Gel Time - Modelo Fishers Johns Melting Point

\section{Caracterização do Material}

Para estudaro efeito do tempo de exposição iniciou-se a preparação dos corpos de provas (cdp's) com o corte do filme em ambiente controlado de temperatura $\left(22^{\circ} \mathrm{C} \pm 3^{\circ} \mathrm{C}\right)$ e umidade relativa do ar $(50 \% \pm 5 \%)$ e submetidos a essa condição ambiente em diferentes intervalos de tempo, contabilizados em "dias de exposição".

A condição do material no momento do corte das amostras foi considerada como "zero dia de exposição". Para o processo de envelhecimento à temperatura ambiente, as amostras foram submetidas a diferentes intervalos de tempo (3, 5, 8, 10 e 12 dias). Ao atingirem o tempo de exposição desejado, as amostras foram embaladas em filme plástico, seladas, identificadas e armazenadas em freezer à $-18^{\circ} \mathrm{C}$ (Tabela 1).

Para verificar o efeito do tempo de exposição e do tempo estocagem do material no freezer simultaneamente, foram preparados 4 conjuntos de amostras (kits), cada um possuindo amostras submetidas a diferentes intervalos de tempo de exposição à temperatura ambiente (3, 5, 8, 10 e 12 dias). Cada kit foi armazenado em freezer por quatro intervalos de tempo diferentes $(0,4,8$ e 12 meses $)$, conforme indicado na Tabela 1 .

Tabela 1. Descrição das amostras ensaiadas por Kit, dias de exposição e tempo de estocagem.

\begin{tabular}{lcccc}
\hline & Kit 1 & Kit 2 & Kit3 & Kit 4 \\
\hline Exposição ambiente (dias) & $3,5,8,10$ e 12 & $3,5,8,10$ e 12 & $3,5,8,10$ e 12 & $3,5,8,10$ e 12 \\
Estocagem em freezer (meses) & 0 & 4 & 8 & 12 \\
\hline
\end{tabular}


Os cdp's preparados foram submetidos aos ensaios de tempo de gelificação, teor de voláteis, capacidade de fluxo, aderência e solubilidade.

O ensaio de tempo de gelificação é baseado na norma ASTM D3532-M ${ }^{[17]}$. Consiste em determinar o tempo em que, a uma determinada temperatura, a resina polimérica passa do estado líquido para o início do estado sólido, determinando a perda da fluidez da matriz.

Para tal, o equipamento Gel Time é aquecido na faixa de temperatura de $166,4^{\circ} \mathrm{C}$ a $177,6^{\circ} \mathrm{C}$. Coloca-se então uma lâmina de vidro sobre o equipamento para aquecimento por 20 segundos. Em seguida adiciona-se a amostra e uma segunda lâmina de vidro. O tempo é cronometrado até o ponto de gelificação da resina, considerado como o momento de transformação para o estado sólido.

Para a análise de teor de voláteis, as amostras foram cortadas nas dimensões de $10 \mathrm{~cm}^{2}$, pesadas em balança analítica e colocadas na estufa na faixa de temperatura de $166,4{ }^{\circ} \mathrm{C}$ a $177,6^{\circ} \mathrm{C}$ por 1 hora. Após este período, foram retiradas da estufa e colocadas em dessecador até atingirem a temperatura ambiente. Uma vez estabilizada a temperatura, são novamente pesadas e calcula-se a perda de massa conforme ASTM D3530/D3530M-97 ${ }^{[18]}$.

O ensaio de capacidade de fluxo foi realizado para determinar o teor de fluxo sob pressão e temperatura controlada $^{[19]}$. Para o ensaio foram cortadas amostras com o diâmetro de $6,5 \mathrm{~cm}$. Um tecido de teflon não poroso foi colocado sobre o adesivo e o conjunto posicionado em prensa aquecido na faixa de temperatura de $166,4^{\circ} \mathrm{C}$ a $177,6^{\circ} \mathrm{C}$ em prensa, durante 1 hora. $\mathrm{O}$ valor de fluxo é expresso em termos de variação percentual de área. Para o presente trabalho, a variação da área foi calculada a partir do diâmetro inicial da amostra e de um diâmetro final médio aproximado (D1), calculado por meio do comprimento (L) e largura máxima(W) resultantes após aquecimento (Figura 2).

A capacidade de aderência é avaliada por meio do contato do material não curado a si mesmo e a uma superfície

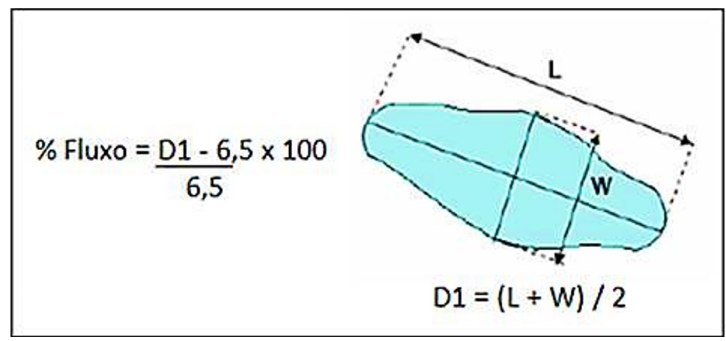

Figura 2. Modelo de medição de área do fluxo, após a cura do adesivo. (L: medida do comprimento máximo, W: medida da largura, essas medidas definem D1) e fórmula para cálculo. metálica posicionada na vertical, em condição ambiente. O ensaio adotado foi baseado na norma NMS $5320^{[20]}$, o qual inicia-se com o corte das amostras nas dimensões de $10 \mathrm{~cm} \times 5 \mathrm{~cm}$. A primeira amostra é fixada em uma chapa de alumínio de $400 \mathrm{~cm}^{2}$ com uma leve pressão exercida por meio de uma espátula plástica. Posteriormente, aplica-se sobre a amostra uma segunda camada para verificar a aderência do filme a si mesmo. A placa é posicionada verticalmente durante 30 minutos. Após esse período, é definido o nível de aderência com base no critério definido na Tabela 2 . A aderência é um fator importante para estes adesivos, pois determina a qualidade da colagem em um substrato ou sobre o próprio adesivo ${ }^{[20]}$.

Não foi encontrado na literatura um método para determinar a solubilidade de adesivos em solvente orgânico, portanto uma metodologia específica foi proposta para avaliar a solubilidade através da extração da resina pelo solvente. Neste caso, foi utilizado um solvente orgânico à base de éster dibásico, conhecido comercialmente como Rhodiasolv (Rhodia), com alto poder de solvência. É um produto não tóxico e não inflamável, biodegradável e de baixo VOC (composto orgânico volátil) ${ }^{[21]}$.

Para a realização do ensaio, as amostras foram cortadas com $50 \mathrm{~cm}^{2}$, em formato retangular $(10 \mathrm{~cm} \times 5 \mathrm{~cm})$, pesadas em balança analítica e imersas uma a uma em um béquer com $300 \mathrm{ml}$ do solvente, com agitação constante, durante 30 segundos. Em seguida, a amostra é retirada e deixada em descanso até a evaporação total do resíduo de solvente, sendo pesada novamente para a realização do cálculo da perda de material.

Os ensaios em DSC foram realizados conforme método dinâmico nas amostras do kit 3, com variação de temperatura de $25^{\circ} \mathrm{C}$ a $350^{\circ} \mathrm{C}$, com uma razão de aquecimento de $10^{\circ} \mathrm{C} / \mathrm{min}$ e atmosfera inerte de $\mathrm{N}_{2}$ com vazão $20 \mathrm{ml} / \mathrm{min}$. Foram utilizados aproximadamente $8,5 \mathrm{mg}$ do material por amostra. Este método de caracterização foi utilizado como técnica auxiliar para avaliar termicamente a cura do filme adesivo. Os parâmetros investigados foram a temperatura onset (Tonset), a temperatura no máximo do pico (Tmáx) e a variação de entalpia $(\Delta \mathrm{E})^{[22]}$

\section{Resultados e Discussão}

A seguir estão apresentados os resultados dos ensaios físico-químicos adotados.

\subsection{Gelificação}

Os valores encontrados por meio deste ensaio (Tabela 3 ) indicam que quanto maior o tempo de exposição, menor o tempo de gelificação do polímero, o que pode ser

Tabela 2. Níveis de Aderência ${ }^{[20]}$

\begin{tabular}{ll}
\hline Nível I & Baixa aderência. Material seco e rígido. \\
Nível II & Baixa aderência, porém maleável \\
Nível III & Leve aderência a si mesmo. Incapaz de aderir à superfície da placa metálica na vertical por mais de 30 minutos; \\
Nível IV & Boa aderência a si mesmo. Capaz de aderir à superfície da placa metálica na vertical por mais de 30 minutos; \\
Nível V & Muita aderência; A resina cola nas luvas. Capaz de aderir a si mesmo e à superfície da placa metálica na vertical por mais de 30 minutos; \\
Nível VI & $\begin{array}{l}\text { Alta aderência. Material "grudento e molhado". A resina cola nas luvas. Existe transferência de resina. Capaz de aderir a si mesmo } \\
\text { e à superfície da placa metálica na vertical por mais de } 30 \text { minutos; }\end{array}$ \\
\hline
\end{tabular}


verificado pela queda gradual e contínua do tempo de gelificação ao longo dos 12 dias de exposição para todos os kits, provavelmente devido a um ligeiro aumento do número de ligações cruzadas do polímero termorrígido ocorrido mesmo à temperatura ambiente. Comparando-se os tempos de gelificação entre os diferentes kit para verificar a eficiência de estocagem do material em freezer, tomando o kit 1(tempo de estocagem igual a 0 meses), observa-se uma redução no tempo de gelificação quando o material é novo (zero dias de exposição)e a partir de 10 dias de exposição à temperatura ambiente ( 10 e 12 dias de exposição) quando comparado aos demais kits. Este processo de reticulação foi evitado durante o período de estocagem em freezer porque não houve alteração significativa nos tempo de gelificação entre os kits 2,3 e 4 .

\subsection{Capacidade de fluxo}

A Tabela 4 apresenta os resultados dos ensaios de capacidade de fluxo para os quatro ciclos de armazenamento.

Apesar do elevado desvio padrão apresentado nos resultados encontrados neste ensaio é possível observar uma diminuição progressiva na capacidade do adesivo em fluir com o aumento do tempo de exposição ao ambiente em todos os kits, sugerindo uma possível reticulação entre as cadeias poliméricas. Assim, essa variação pode ser considerada como inerente à metodologia de ensaio. Comparando-se os resultados entre os kits não é possível afirmar que o período de estocagem tenha influenciado na capacidade de fluxo do material.

Na Figura 3 estão apresentados os cdp's resultantes do ensaio para as amostras do Kit 1. Pode-se observar claramente a redução da capacidade de fluxo pela redução da área após o escoamento da resina por meio da modificação das variáveis, $\mathrm{L}$ (medida do comprimento máximo), $\mathrm{W}$ (medida da largura), e consequentemente a variação de D1.

\subsection{Aderência}

Os resultados dos ensaios de aderência para os quatro ciclos de armazenamento estão apresentados na Tabela 5:

Pode-se observar que para as amostras do kit 1 , nos períodos de exposição iniciais (0 e 3 dias) não houve modificação considerável de aderência sendo notado variação

Tabela 3. Tempos de gelificação (min).

\begin{tabular}{ccccc}
\hline Exposição (dias) & Kit 1 & Kit 2 & Kit3 & Kit 4 \\
\hline $\mathbf{0}$ & $13,82 \pm 0,55$ & $11,12 \pm 0,18$ & $10,55 \pm 0,57$ & $10,08 \pm 0,3$ \\
$\mathbf{3}$ & $9,23 \pm 0,25$ & $9,53 \pm 0,23$ & $9,72 \pm 0,37$ & $9,1 \pm 0,30$ \\
$\mathbf{5}$ & $8,53 \pm 0,35$ & $8,6 \pm 0,15$ & $8,88 \pm 0,32$ \\
$\mathbf{8}$ & $8,22 \pm 0,15$ & $8,42 \pm 0,12$ & $5,87 \pm 0,20$ & $5,27 \pm 0,55$ \\
$\mathbf{1 0}$ & $7,58 \pm 0,22$ & $5,83 \pm 0,22$ & $3,05 \pm 0,60$ & $6,00 \pm 33 \mathrm{~s}$ \\
\hline $\mathbf{1 2}$ & $5,93 \pm 0,25$ & $2,98 \pm 0,17$ & $2,98 \pm 0,32$ \\
\hline
\end{tabular}

Tabela 4. Capacidade de fluxo (\%).

\begin{tabular}{|c|c|c|c|c|}
\hline Exposição (dias) & Kit 1 & Kit 2 & Kit 3 & Kit 4 \\
\hline 0 & $399 \pm 14$ & $368 \pm 14$ & $391 \pm 7$ & $367 \pm 32$ \\
\hline 3 & $345 \pm 15$ & $330 \pm 14$ & $343 \pm 25$ & $345 \pm 25$ \\
\hline 5 & $299 \pm 16$ & $268 \pm 20$ & $270 \pm 36$ & $311 \pm 6$ \\
\hline 8 & $155 \pm 4$ & $153 \pm 8$ & $201 \pm 4$ & $273 \pm 31$ \\
\hline 10 & $157 \pm 4$ & $117 \pm 6$ & $154 \pm 40$ & $215 \pm 72$ \\
\hline 12 & $76 \pm 6$ & $77 \pm 11$ & $78 \pm 7$ & $93 \pm 10$ \\
\hline
\end{tabular}
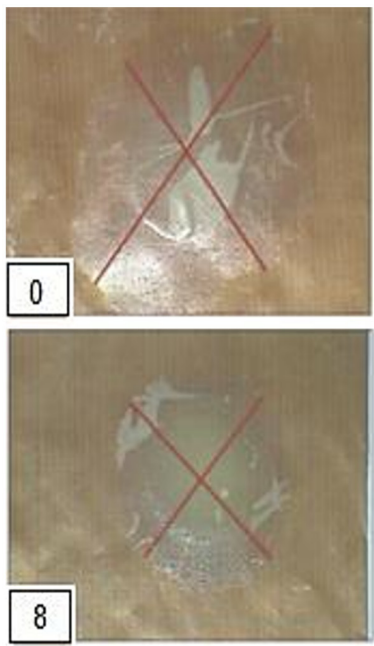
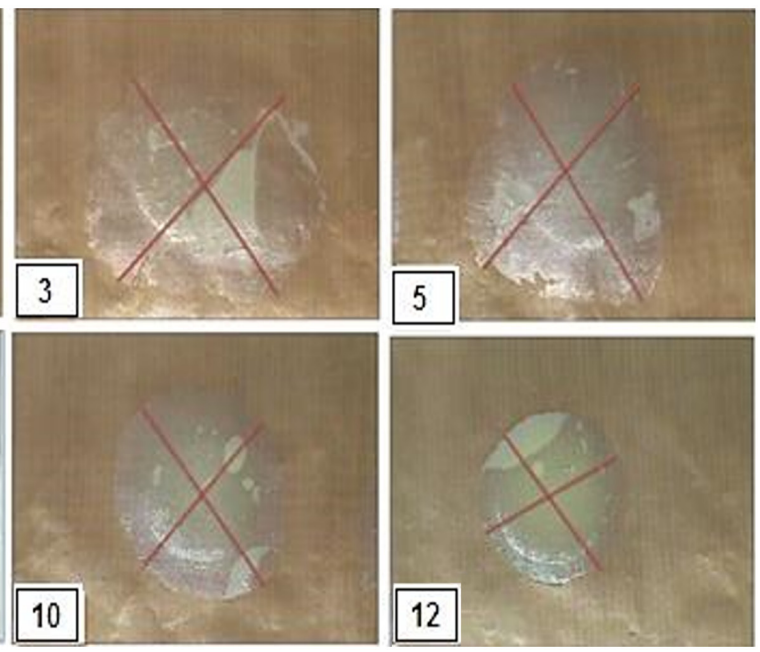

Figura 3. Fotos do ensaio de fluxo, o número indicado em cada foto corresponde ao período de envelhecimento de cada material em dias. 
detectável de aderência (de nível IV para III) apenas após o quinto dia de exposição, permanecendo nesse nível até 12 dias. Quando comparados os dados referentes aos tempos de exposição e de armazenamento, nota-se certa influência também do tempo de armazenamento nessa propriedade o que indica um provável enrijecimento destes adesivos. Parte desse efeito devido ao tempo de armazenamento pode ter sido causado por possível absorção de umidade como insinuado pelos resultados de teor de voláteis. Embora essa relação não tenha sido verificada, essa possibilidade está indicada em literatura ${ }^{[4]}$.

\subsection{Teor de voláteis}

A tabela 6 apresenta os resultados dos ensaios de teor de voláteis médios para os quatro ciclos de exposição e armazenamento. Apesar da metodologia indicar uma faixa de variação de temperatura larga $\left(166,4^{\circ} \mathrm{C}\right.$ a $\left.177,6^{\circ} \mathrm{C}\right) \mathrm{o}$ desvio-padrão dos resultados indica uma boa repetibilidade dos resultados encontrados, com exceção da amostra de 3 dias de exposição do kit 2 .

Conforme ocorre o aumento do período de exposição, os adesivos apresentaram um aumento no teor de voláteis ao longo dos 12 dias. Embora a causa não tenha sido investigada, estima-se que este aumento possa ter ocorrido devido à absorção de umidade. Mesmo os materiais estando embalados durante o período de armazenamento em freezer, o aumento de teor de voláteis é significativo entre os kits 1 a 3, após este período a absorção de umidade é estabilizada (kit 4). Este aumento de voláteis credita-se ao fato da matriz de resinas epoxídicas e do reforço de poliamida serem intrinsecamente higroscópicos.

\subsection{Solubilidade}

A Tabela 7 apresenta os resultados dos ensaios de solubilidade em solvente orgânico.

Os resultados demonstram que o período de exposição ao ambiente tem grande influência na solubilidade da resina, sendo que o tempo de armazenamento não apresentou a mesma influência para essa propriedade.

A diminuição da extração da resina pela presença de solvente indica uma redução da solubilidade desta após o tempo de exposição. Conforme aumenta o tempo de exposição do adesivo, a solubilidade da resina no solvente apresenta redução consistente. Lucas e co-autores explicam que esses aglomerados ocorrem pelo empacotamento das cadeias dificultando a difusão do solvente no material e que, quanto menor a distância intermolecular, menos a resina se solubilizará ${ }^{[22]}$.

$\mathrm{O}$ aspecto dos adesivos, apresentado na Figura 4, se repetiu para todos os ciclos ensaiados, onde a figura representativa de 0 dia de exposição apresentou apenas fibras após a extração, e no outro extremo, com 12 dias de exposição, observa-se grande quantidade de resina não removida pelo solvente, evidenciando assim a perda de solubilidade. Nos períodos intermediários, notou-se diminuição constante da solubilidade, por meio da remoção parcial de resina em

Tabela 5. Ensaio de aderência (nível).

\begin{tabular}{ccccc}
\hline Exposição (dias) & Kit 1 & Kit 2 & Kit 3 & Kit 4 \\
\hline $\mathbf{0}$ & IV & III & III & III \\
$\mathbf{3}$ & IV & III & III \\
$\mathbf{5}$ & III & III & III & III \\
$\mathbf{8}$ & III & III & II & II \\
$\mathbf{1 0}$ & III & III & II & II \\
\hline 12 & III & II & II \\
\hline
\end{tabular}

Tabela 6. Teor de voláteis (\%).

\begin{tabular}{ccccc}
\hline Exposição (dias) & Kit 1 & Kit 2 & Kit 3 \\
\hline $\mathbf{0}$ & $0,68 \pm 0,01$ & $1,35 \pm 0,02$ & $2,01 \pm 0,01$ & $2,00 \pm 0,03$ \\
$\mathbf{3}$ & $0,67 \pm 0,02$ & $1,10 \pm 0,37$ & $2,06 \pm 0,03$ & $2,04 \pm 0,03$ \\
$\mathbf{5}$ & $0,75 \pm 0,04$ & $1,26 \pm 0,06$ & $2,05 \pm 0,02$ & $2,03 \pm 0,02$ \\
$\mathbf{8}$ & $0,74 \pm 0,01$ & $1,27 \pm 0,02$ & $2,12 \pm 0,02$ \\
$\mathbf{1 0}$ & $1,10 \pm 0,05$ & $1,30 \pm 0,01$ & $2,02 \pm 0,02$ \\
$\mathbf{1 2}$ & $1,27 \pm 0,03$ & $1,30 \pm 0,01$ & $2,10 \pm 0,02 \pm 0,02$ \\
\hline
\end{tabular}

Tabela 7. Solubilidade (\%).

\begin{tabular}{ccccc}
\hline Exposição (dias) & Kit 1 & Kit 2 & Kit 3 & Kit 4 \\
\hline $\mathbf{0}$ & $95 \pm 1$ & $96 \pm 1$ & $93 \pm 2$ & $93 \pm 3$ \\
$\mathbf{3}$ & $93^{*}$ & $94 \pm 1$ & $88 \pm 3$ \\
$\mathbf{5}$ & $87 \pm 2$ & $87^{*}$ & $85 \pm 2$ \\
$\mathbf{8}$ & $85 \pm 1$ & $86 \pm 1$ & $85 \pm 4$ \\
$\mathbf{1 0}$ & $75 \pm 2$ & $72 \pm 1$ & $81 \pm 2$ & $85 \pm 2$ \\
$\mathbf{1 2}$ & $74 \pm 2$ & $69 \pm 1$ & $77 \pm 2$ & $73 \pm 2$ \\
\hline
\end{tabular}

*Os resultados apresentaram desvio-padrão abaixo do número de algarismos significativo adotados para o ensaio. 

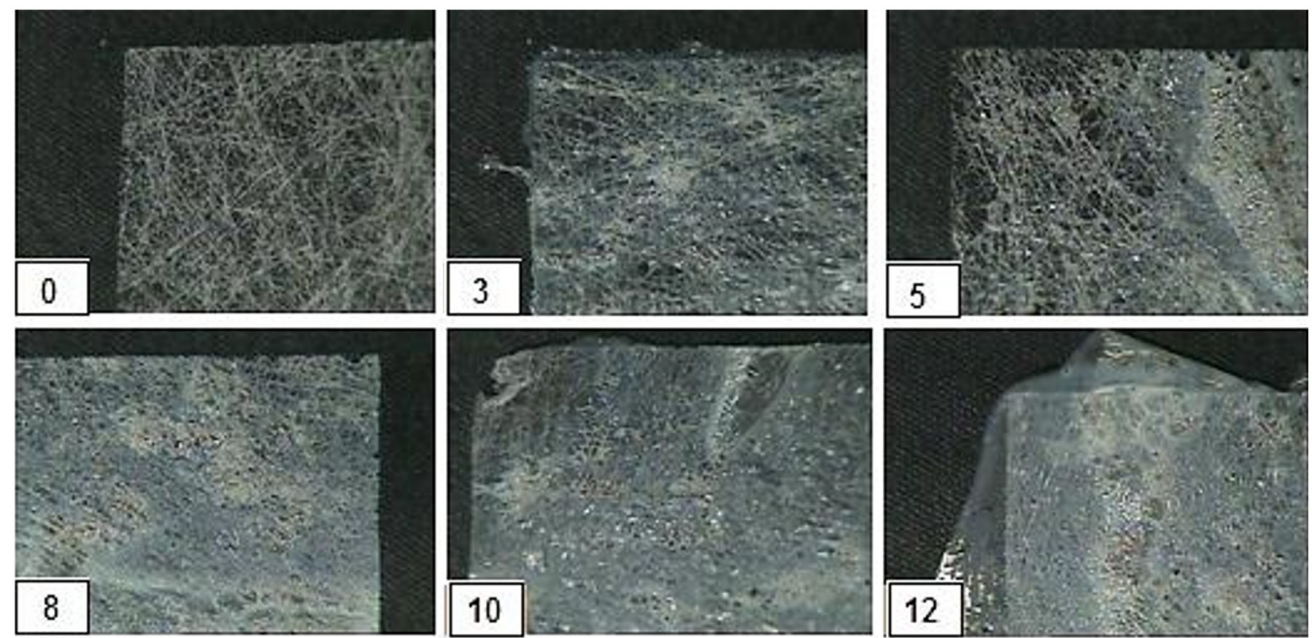

Figura 4. Fotos do ensaio de solubilidade após extração da resina com solvente orgânico, o número indicado em cada foto corresponde ao período de envelhecimento de cada material em dias.

Tabela 8. Dados obtidos da análise de Calorimetria Exploratória Diferencial (DSC) para o Kit no 3 .

\begin{tabular}{cccc}
\hline Exposição (dias) & Temperatura Onset $\left({ }^{\circ} \mathbf{C}\right)$ & Temperatura de Pico $\left({ }^{\circ} \mathbf{C}\right)$ & Entalpia $\Delta \mathbf{E}(\mathbf{J} / \mathbf{g})$ \\
\hline $\mathbf{0}$ & 191 & 227 & 258 \\
$\mathbf{3}$ & 187 & 229 & 301 \\
$\mathbf{5}$ & 188 & 230 & 294 \\
$\mathbf{8}$ & 196 & 229 & 239 \\
$\mathbf{1 0}$ & 190 & 229 & 227 \\
$\mathbf{1 2}$ & 191 & 230 & 239 \\
\hline
\end{tabular}

quantidades cada vez menores. Estes resultados indicam que houve um aumento do número de reticulações no material termorrígido, que mesmo em baixas concentrações, dificulta a difusão do solvente dentro da massa polimérica.

\subsection{Aspecto visual}

Observando-se as amostras a olho nú, antes dos ensaios, notou-se que, com 0 dia de exposição à temperatura ambiente, estas apresentavam uma superfície lisa, homogênea e brilhante. Para as amostras entre 3 e 5 dias de exposição, o adesivo já não era mais tão liso, apresentando também redução na homogeneidade e no brilho. Para os materiais entre 8 e 12 dias o material apresento-se rugoso, fosco e quebradiço.

\subsection{Calorimetria Exploratória Diferencial (DSC)}

A partir dos resultados das curvas de DSC, nos diferentes tempos de exposição apresentados na Tabela 8 , obtidos a partir das amostras do kit 3, foi possível observar que as variáveis Tonset, Tmáx e $\Delta \mathrm{E}$ não apresentaram variações coerentes ou mudanças significativas na cura da resina relacionadas ao armazenamento e/ou envelhecimento à temperatura ambiente (Figura 5). Estes resultados sugerem que o possível aumento no número de reticulações da matriz polimérica à temperatura ambiente, não foi comprovado por este método de análise térmica, já que não houve alteração significativa da entalpia de cura ao longo do período de exposição, o que pode ser atribuído a uma taxa de cura muito baixa da matriz ou apenas pela falta sensibilidade do método.

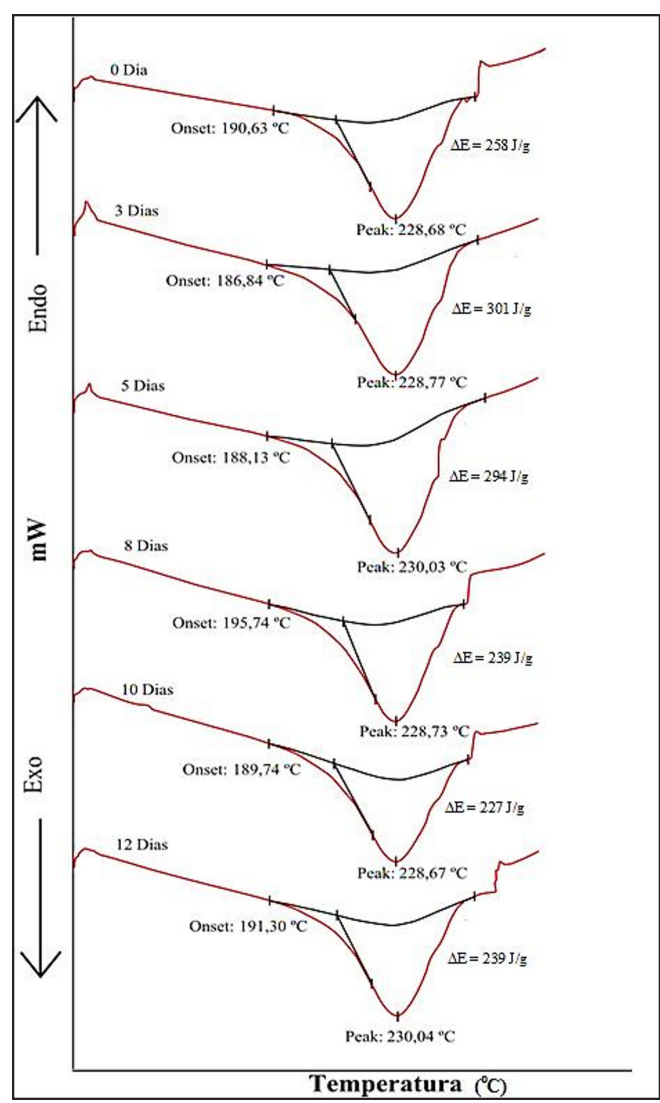

Figura 5. Calorimétrica exploratória diferencial (DSC). 


\section{Conclusão}

De acordo com os resultados apresentados, independente do ciclo analisado, as amostras com 0 dia de envelhecimento, ou seja, adesivo com baixo tempo de exposição, apresentou elevada solubilidade da resina no solvente, elevada capacidade de fluir e altas propriedades de aderência dentro da escala adotada. Entre os materiais com 3 e 5 dias de envelhecimento, o adesivo apresentou uma pequena defasagem nos resultados quando comparados com os materiais de 0 dia. Neste caso, o material se encontra com o "tack" inferior, porém ainda apresenta boas características de aderência.

Observa-se que no período de envelhecimento entre 5 e 8 dias o material sofreu um decréscimo na capacidade de fluxo e aderência, agravando-se conforme o aumento do tempo de exposição à temperatura ambiente. Nas propriedades de aderência e principalmente na solubilidade, observou-se visualmente que a resina aglomerou-se nas fibras de nylon e a matriz não flui mais de forma homogênea.

Nos ensaios entre 10 e 12 dias de exposição à temperatura ambiente foi observado que os materiais apresentaram aparência fosca, pouca ou nenhuma aderência e a resina se aglomerou quase que totalmente nas fibras de nylon.

$\mathrm{O}$ teste de solubilidade apresentou boa relação com o decréscimo de outras propriedades. Embora não se possa fazer uma correlação numérica direta, o teste de solubilidade mostrou-se sensível à transformação do material demonstrando que, por se tratar de um parâmetro de avaliação rápido, simples e barato, sem a necessidade de equipamentos auxiliares, é um instrumento potencial de avaliação muito forte para uso no meio produtivo.

Finalmente, conclui-se que o a exposição à temperatura ambiente tem efeito deletério generalizado nas propriedades avaliadas, mas quando armazenados à baixa temperatura, mesmo por tempo exponencialmente superior aos avaliados em temperatura ambiente, as propriedades sofreram pouca ou alteração não detectável.

Assim, a exposição prolongada de materiais não curados às condições de temperatura e umidade acima das recomendadas pelo fabricante contribuem na redução do desempenho e podem se tornar causas potenciais de falha prematura da ligação das superfícies unidas por adesivos.

Para o futuro, sugere-se uma análise mais detalhada sobre o assunto, inclusive com outros sistemas de resinas para avaliar a dependência dessas propriedades com relação à formulação. Também é interessante aumentar o período de armazenamento para verificar a transformação nessa condição e tentar correlacionar com dados de envelhecimento ao ambiente de forma a utilizar este último como ensaio acelerado.

Sugere-se ainda a complementação do estudo com outras técnicas para avaliar o grau de cura da matriz polimérica e ensaios mecânicos para avaliação do impacto das transformações detectadas nas propriedades finais de colagem.

\section{Referências}

1. American Society for Testing and Materials-ASTM. (2011). ASTM D907: standard terminology of adhesives. West Conshohocken: ASTM.
2. Sharpe, L. H. (1990). Section1. Fundamentals of adhesives and sealants technology. In ASM. Engineered materials handbook - adhesives and sealants (Vol. 3, pp. 1-72). Materials Park: ASM International.

3. Hexcel. (2003). Handbook Redux $®$ Bonding Technology. Stamford: Hexcel Corporation. Recuperado em 10 de setembro de 2013, de http://www.hexcel.com/Resources/DataSheets/ Brochure-Data-Sheets/Adhesive_Bonding_Technology.pdf

4. Petrie, E. M. (2007). Handbook of adhesives and selants (2. ed). New York: The McGraw-Hill Companies.

5. Campbell, F. C. (2006). Manufacturing technology for aerospace structural materials. Amsterdam: Elsevier Science.

6. Schwartz, M. M. (1997). Composite materials: properties, nondestructive testing, and repair. New Jersey: Prentice-Hall Inc.

7. Rezende, M. C., Botelho, E. C., Paiva, J. M. F., \& Costa, M. L. (2003). Avaliação Térmica e reológica do ciclo de cura do pré-impregnado de carbono epóxi. Polímeros: Ciência e Tecnologia, 13(3), 188-197. http://dx.doi.org/10.1590/S010414282003000300009.

8. Callister, W.D., Jr., \& Rethwisch, D. G. (1991). Materials science and engineering an introduction. New York: John Wiley \& Sons.

9. Fraga, F., Castro-Díaz, C., Rodriguez-Nuñez, E., \& MartínezAgeitos, J. M. (2003). Physical aging for an epoxy network diglycidyl ether of bisphenol A/m-xylylenediamine. Polymer, 44(19), 5779-5784. http://dx.doi.org/10.1016/S00323861(03)00624-4.

10. Riegel, I. C., Freitas, L. L., \& Samios, D. (1999). Envelhecimento físico de sistemas DGEBA/DDM investigado por análise térmica (DSC/DMA). Polimeros: Ciência e Tecnologia, 9(3), 58-64. http://dx.doi.org/10.1590/S0104-14281999000300011.

11. Kim, D., Centea, T., \& Nutt, R. (2014). Out-time effects on cure kinetics and viscosity for an out-of-autoclave (OOA) prepreg: Modelling and monitoring. Composites Science and Technology, 100, 63-69. http://dx.doi.org/10.1016/j. compscitech.2014.05.027.

12. Sales, R. C. M., \& Dibbern-Brunelli, D. (2008). Efeito higrotérmico em prepregs de fibra de vidro/epóxi por espectroscopia de luminescência. Polímeros: Ciência e Tecnologia, 18(1), 52-56. http://dx.doi.org/10.1590/S0104-14282008000100011.

13. Yagoubi, J. E., Lubineau, G., Traidia, A., \& Verdu, J. (2015). Monitoring and simulations of hydrolysis in epoxy matrix composites during hygrothermal aging. Composites. Part A, Applied Science and Manufacturing, 68, 184-192. http://dx.doi. org/10.1016/j.compositesa.2014.10.002.

14. Ray, B. C. (2006). Temperature effect during humid ageing on interfaces of glass and carbon fibers reinforced epoxy composites. Journal of Colloid and Interface Science, 298(1), 111-117. http://dx.doi.org/10.1016/j.jcis.2005.12.023. PMid:16386268.

15. Sales, R. C. M., Diniz, M. F., Dutra, R. C. L., Thim, G. P., \& Dibbern-Brunelli, D. (2010). Thermal curing of glass-epoxy prepregs by luminescence spectroscopy. Journal of Applied Polymer Science, 117(2), 664-671. http://dx.doi.org/10.1002/ app.31953.

16. Twisk, J. V., \& Aker, S. C. (1990). Storing adhesives and sealant materials. In ASM. Engineered materials handbookadhesives and sealants (Vol. 3, pp. 683-686). Materials Park: ASM International.

17. American Society for Testing and Materials-ASTM. (1999). ASTM D3532/D3532M: gel time of carbon fiber-epoxy prepreg. West Conshohocken: ASTM.

18. American Society for Testing and Materials - ASTM. (1997). ASTM D3530/D3530-M: volatiles content of composite material prepreg. West Conshohocken: ASTM. 
19. American Society for Testing and Materials-ASTM. (2011). ASTM D3531/D3531-M: resin flow of carbon fiber-epoxy prepreg. West Conshohocken: ASTM.

20. National Center for Advanced Materials Performance - NCAMP. (2015). NMS 5320: material specification. Wichita: National Institute for Aviation Research.

21. Rhodia Solvay Group. (2013). Rhodiasolv ${ }^{\circledR}$ PolarClean. Solvay. Recuperado em 09 de setembro de 2013, de http://www. rhodia.com.br/pt/mercados-e-produtos/catalogo-de-produtos/ Rhodiasolv-PolarClean.html

22. Lucas, E. F., Soares, B. G., \& Monteiro, E. E. C. (2001). Caracterização de polímeros: determinação de peso molecular e análise térmica. Rio de Janeiro: E-papers.

Enviado: Set. 17, 2014 Reenviado: Ago. 28, 2015 Aceito: Set. 08, 2015 